\title{
The Functional Dysphagia Scale Is a Useful Tool for Predicting Aspiration Pneumonia in Patients With Parkinson Disease
}

\author{
Jong Hwa Lee, MD ${ }^{1}$, Kyeong Woo Lee, MD¹, Sang Beom Kim, MD ${ }^{1}$, \\ Sook Joung Lee, $\mathrm{MD}^{1}$, Sang Myung Chun, $\mathrm{MD}^{2}$, Sung Moon Jung, $\mathrm{MD}^{1}$
}

Departments of ${ }^{1}$ Physical Medicine and Rehabilitation, ${ }^{2}$ Neurology, Dong-A University College of Medicine, Busan, Korea

\begin{abstract}
Objective To describe the correlation between the functional dysphagia scale and aspiration pneumonia and which characteristics influence the occurrence of aspiration pneumonia in patients with idiopathic Parkinson disease.

Methods Fifty-three patients with idiopathic Parkinson disease were prospectively evaluated in this study. Disease severity and functional status were measured by modified Hoehn and Yahr (H\&Y) staging, Schwab and England activities of daily living (S-E ADL) scale and Korean version of Mini-Mental State Examination (K-MMSE). Swallowing function was evaluated by the functional dysphagia scale (FDS) and the penetration-aspiration scale (PAS) based on a videofluoroscopic swallowing study. The patients were followed up for 3 months and divided into two groups according to the occurrence of aspiration pneumonia. The correlation between the variables and aspiration pneumonia was analyzed.

Results Eight patients of the 53 patients were allocated to the aspiration pneumonia group and 45 patients to the non-aspiration pneumonia group. The patients in the aspiration pneumonia group had significantly higher H\&Y staging, and scored lower on S-E ADL scale and K-MMSE. The patients in the aspiration pneumonia group had significantly higher scores on FDS and PAS. A multiple logistic regression analysis showed that the S-E ADL scale and the FDS were associated with the occurrence of aspiration pneumonia in the patients with Parkinson disease. Conclusion Given that the FDS can quantitatively assess the functional problems associated with dysphagia, it can be clinically effective in predicting the occurrence of aspiration pneumonia, and the FDS and the S-E ADL scale could be predictive variables for aspiration pneumonia in patients with Parkinson disease.
\end{abstract}

Keywords Parkinson disease, Dysphagia, Swallowing disorders, Fluoroscopy

Received June 29, 2015; Accepted October 14, 2015

Corresponding author: Sung Moon Jung

Department of Physical Medicine and Rehabilitation, Dong-A University College of Medicine, 26 Daesingongwon-ro, Seo-gu, Busan 49201, Korea. Tel: +82-51-240-5690, Fax: +82-51-254-8511, E-mail: zzugul36@naver.com

ORCID: Jong Hwa Lee (http://orcid.org/0000-0003-2489-358X); Kyeong Woo Lee (http://orcid.org/0000-0001-8785-5535); Sang Beom Kim (http:// orcid.org/0000-0002-5622-5933); Sook Joung Lee (http://orcid.org/0000-0002-6894-445X); Sang Myung Chun (http://orcid.org/0000-0001-5905-2188); Sung Moon Jung (http://orcid.org/0000-0003-2674-5337).

() This is an open-access article distributed under the terms of the Creative Commons Attribution Non-Commercial License (http://creativecommons.org/ licenses/by-nc/4.0) which permits unrestricted noncommercial use, distribution, and reproduction in any medium, provided the original work is properly cited. Copyright $\odot 2016$ by Korean Academy of Rehabilitation Medicine 


\section{INTRODUCTION}

Parkinson disease is a representative neurodegenerative disease that occurs as dopamine production in the substantia nigra gradually diminishes, resulting in tremors, bradykinesia, rigidity, and unstable posture $[1,2]$. In addition to difficulty with daily activities, dysphagia also occurs frequently among patients with Parkinson disease [3-5]. This in turn causes aspiration pneumonia, which is one of the major causes of death among patients with Parkinson disease [6-11]. A variety of other factors, including functional conditions, oral hygiene, and accompanying diseases, may also bring about the occurrence of aspiration pneumonia among patients with Parkinson disease [12-15].

As with dysphagia, patients in the early stages of Parkinson disease often complain of difficulty in starting swallowing and eating due to mobility dysfunction, insufficient movement of saliva and food in the oral cavity, and labored lingual movements [16]. Furthermore, abnormal patterns of swallowing are observed, such as problems with epiglottic motion, slow recovery of the pre-swallow resting position, and delayed transfer and stasis of food [17-19]. An increase in the oral transit time and tongue pumping [20], are frequently reported among the secondary symptoms relevant to the disease, along with bradykinesia and rigidity $[17,21]$.

The videofluoroscopic swallowing study (VFSS) is a standard tool for evaluating abnormalities in swallowing. With this test the risks of aspiration and complications with respiratory devices can be assessed. In addition, revisions to diet and compensatory strategies during swallowing can be established. Previous literature has reported that a high percentage of patients with Parkinson disease have a swallowing disability based on VFSS results [22]. Several studies using the VFSS with visualperceptual parameters have evaluated the abnormalities of patients with Parkinson disease in the oral and pharyngeal phases. However, it is challenging to verify the pathological mechanism of dysphagia among patients with Parkinson disease using spatial and time variables [18]. Moreover, only a small portion of studies have discussed the relationship between these factors and aspiration pneumonia.

Some researchers have examined the relevance of aspiration by evaluating the functional dysphagia scale (FDS) scores of patients with stroke through the VFSS [23]. This scale is used to quantify functional dysphagia, such as oral movement, the defense mechanisms of the pharynx, and the amount of residue after swallowing. Few studies have applied this method to patients with Parkinson disease.

Therefore, the purpose of this study is to investigate which parameters and characteristics influence the occurrence of aspiration pneumonia, and to describe the correlation between the functional dysphagia scale and aspiration pneumonia in patients with Parkinson disease.

\section{MATERIALS AND METHODS}

\section{Research subjects}

Sixty-six patients with idiopathic Parkinson disease who were hospitalized in or visited the outpatient clinic at the Department of Physical Medicine and Rehabilitation and the Department of Neurology at Dong-A University Hospital from January 2013 to December 2014 were included in this study. The study only included patients on the regular, blend or soft oral diets. Patients with secondary Parkinson disease related to drugs, or infection that could cause fluctuating symptoms and neurodegenerative Parkinson syndrome (progressive supranuclear palsy, multiple system atrophy) were excluded. Those with other neurological defects or severe medical diseases which can influence dysphagia, such as stroke, dementia, congestive heart failure, chronic obstructive pulmonary disease, bronchiectasis, and having a tracheostomy were also excluded.

The present prospective study was approved by the Institutional Review Board of Dong-A University Hospital.

\section{Evaluation method}

The functional status and disease severity of all patients was investigated using the Schwab and England activities of daily living (S-E ADL) scale and modified Hoehn and Yahr (H\&Y) staging [24]. The S-E ADL scale is a quantitative tool for evaluating the function of patients with Parkinson disease, where a higher score means a better functional status [24]. The modified H\&Y staging assesses the symptoms of Parkinson disease by degrees from 0 to 5 to indicate the progress of the disease [24]. Furthermore, cognitive function was evaluated using the Korean version of Mini-Mental State Examination (K-MMSE). 
The occurrence of aspiration pneumonia in subjects was followed for 3 months after the initial evaluation. Aspiration pneumonia was diagnosed by the following characteristics: fever accompanied by an increase in leukocytes, abnormal lung sounds, and abnormal findings in a chest $\mathrm{X}$-ray.

\section{Videofluoroscopic swallowing study}

Each subject received the identical VFSS test with the modified Logemann's protocol [25]. This test was performed by experienced doctors in a fluoroscopic X-ray room in the Department of Physical Medicine and Rehabilitation. The subject sat laterally in front of the fluoroscopic tester and took a posture that is appropriate for a 4- to 5-second observation of anatomic structure, during which mouth and lip closure, and masticatory function and cooperation were briefly checked. The study subjects twice ate each of the following: soft blended food, normal regular food with standardized viscosity, and materials that were mixed with a liquid barium solution (barium sulfate; Solotop suspension). Afterward, the subjects were required to drink $100 \mathrm{~mL}$ of liquid barium that was diluted with water at a ratio of $1: 3$ so that invasion and aspiration could be assessed. A video recording was made of the overall test scene. The video was evaluated by three rehabilitation physicians and Rehabilitation who determined the penetration-aspiration score (PAS) and the FDS score. Each subject received the test during a 'drugon' phase, that is, within 90-120 minutes of taking their anti-Parkinson disease medicine.

\section{VFSS analysis}

\section{Functional dysphagia scale}

To quantify the VFSS test results the FDS devised by Han et al. [23] was used, and the lip closure, food bolus formation, and masticatory function in the oral phase were assessed against three levels. The residue inside the oral cavity after swallowing was assessed against four levels. The transit time for what was thought to be the main food bolus during swallowing was measured. During the pharyngeal phase, laryngeal elevation and the decrease in epiglottic closing function, the delay in swallowing reflex, and the barium coating on the pharyngeal wall after swallowing were evaluated. Moreover, the amount of food bolus invasion into the nasal cavity, the amount of residue in the pyriform sinuses after swallowing, and the amount of residue in the vallecular fossa after swallowing were evaluated for classification on one of four levels. The delay in pharyngeal passing time was measured as the time required for the food bolus to pass the cricopharyngeal level from the lower corner of the mandible.

\section{Penetration-aspiration scale}

The PAS [26] is a tool used to evaluate tracheal invasion by a food bolus or liquid. It evaluates the swallowing function by classifying the symptoms that follow into one of eight levels, where a higher score means a poorer swallowing function.

\section{Statistical analysis}

SPSS ver. 18.0 for Windows software (SPSS Inc., Chicago, IL, USA) was used for statistical analysis in this study. The clinical characteristics and VFSS scores of the patients with Parkinson disease were analyzed with a nonparametric testing method using the Mann-Whitney $\mathrm{U}$ test. Patients were divided into two groups, one with aspiration pneumonia and one without aspiration pneumonia. Multiple logistic regressions were performed to control the gender, age, and other statistically significant variables to exclude the effects of factors that might have influenced the occurrence of aspiration pneumonia among the patients with Parkinson disease. A p-value of less than 0.05 was considered significant.

To calculate the sample size for logistic regression analysis, we used $G^{*}$ Power for Windows (ver. 3.1.9) with an effect size of 0.5 , an alpha risk of 0.05 , and a power of 0.80 . The resulting calculated sample size was 53 patients. Considering a $10 \%$ dropout rate, the corrected sample size was 59 patients.

\section{RESULTS}

The VFSS was performed in 66 patients with idiopathic Parkinson disease. Thirteen subjects (19.6\%) with insufficient medical records, loss of follow-up, or other neurologic disease diagnosed in follow-up imaging studies were excluded. During the 3-month follow-up of 53 patients, $8(15.0 \%)$ patients had aspiration pneumonia and $45(84.9 \%)$ patients did not. In the evaluation of disease staging, function, and cognition among the study subjects, the group with aspiration pneumonia showed significantly higher $\mathrm{H} \& \mathrm{Y}$ staging ( $3.70 \pm 0.98$ vs. $1.84 \pm 0.43$; 
Table 1. Characteristics of both groups

\begin{tabular}{lccc}
\hline & $\begin{array}{c}\text { Patients with } \\
\text { aspiration pneumonia (n=8) }\end{array}$ & $\begin{array}{c}\text { Patients without } \\
\text { aspiration pneumonia (n=45) }\end{array}$ & p-value \\
\hline Age & $76.45 \pm 6.59$ & $68.52 \pm 10.21$ & 0.530 \\
Sex (male:female) & $5: 3$ & $23: 22$ & 0.378 \\
Duration (yr) & $8.23 \pm 5.18$ & $4.13 \pm 3.78$ & 0.086 \\
K-MMSE & $21.50 \pm 4.17$ & $26.40 \pm 3.87$ & $0.048^{*}$ \\
H\&Y & $3.70 \pm 0.98$ & $1.84 \pm 0.43$ & $0.004^{*}$ \\
S-E ADL & $52.00 \pm 16.43$ & $88.00 \pm 10.33$ & $0.005^{*}$ \\
\hline
\end{tabular}

Values are presented as mean \pm standard deviation.

K-MMSE, Korean version of Mini-Mental State Examination; H\&Y, modified Hoehn \& Yahr staging; S-E ADL, Schwab and England activities of daily living scale.

${ }^{*} \mathrm{p}<0.05$ by Mann-Whitney U test.

Table 2. Dysphagia scale based on VFSS findings

\begin{tabular}{|lccc}
\hline & $\begin{array}{c}\text { Patients with } \\
\text { aspiration pneumonia } \\
(\mathbf{n = 8})\end{array}$ & $\begin{array}{c}\text { Patients without } \\
\text { aspiration pneumonia } \\
(\mathbf{n}=\mathbf{4 5})\end{array}$ & p-value \\
\hline PAS & $5.37 \pm 2.82$ & $2.23 \pm 1.84$ & $0.047^{*}$ \\
\hline FDS & $29.25 \pm 19.06$ & $6.76 \pm 9.49$ & $0.009^{*}$ \\
\hline Oral phase & $3.50 \pm 5.18$ & $0.00 \pm 0.00$ & 0.093 \\
\hline Pharyngeal phase & $23.75 \pm 19.52$ & $6.76 \pm 9.49$ & $0.021^{*}$ \\
\hline Reduced laryngeal elevation and epiglottic closure & $7.50 \pm 6.21$ & $3.57 \pm 5.54$ & 0.144 \\
\hline Triggering of pharyngeal swallow delay & $3.75 \pm 5.17$ & $0.21 \pm 1.45$ & 0.114 \\
\hline Nasal penetration & $0.00 \pm 0.00$ & $0.00 \pm 0.00$ & 1.000 \\
\hline Residue in piriformis sinuses & $4.00 \pm 3.70$ & $1.19 \pm 2.19$ & $0.048^{*}$ \\
\hline Residue in valleculae & $2.50 \pm 2.97$ & $1.19 \pm 2.02$ & 0.237 \\
\hline Coating of pharyngeal wall after swallow & $5.00 \pm 5.34$ & $0.42 \pm 2.04$ & $0.040^{*}$ \\
\hline Pharyngeal transit time & $2.00 \pm 2.13$ & $0.08 \pm 0.58$ & $0.031^{*}$ \\
\hline
\end{tabular}

Values are presented as mean \pm standard deviation.

VFSS, videofluoroscopic swallowing study; PAS, penetration-aspiration scale; FDS, functional dysphagia scale; oral phase, include section of FDS (lip closure, bolus formation, residue in the oral cavity, oral transit time); pharyngeal phase, include section of FDS (reduced laryngeal elevation and epiglottic closure, triggering of pharyngeal swallow delay, residue in the vallecular fossa, residue in the pyriform sinuses, coating of pharyngeal wall after swallow, pharyngeal transit time).

* $\mathrm{p}<0.05$ by Mann-Whitney U test.

$\mathrm{p}=0.004)$, a lower S-E ADL scale (52.00 \pm 16.43 vs. $88.00 \pm$ 10.33; $\mathrm{p}=0.005)$, and a significantly lower K-MMSE score $(21.50 \pm 4.17$ vs. $26.40 \pm 3.87 ; \mathrm{p}=0.048)$ than the group without aspiration pneumonia. The differences in gender and age between the two groups were not statistically significant (Table 1).

Based on the VFSS results, the group with aspiration pneumonia also showed significantly higher values of FDS (29.25 \pm 19.06 vs. $6.76 \pm 9.49 ; \mathrm{p}=0.009)$ and PAS (5.37 \pm 2.82 vs. $2.23 \pm 1.84 ; \mathrm{p}=0.047$ ) than the group without
Table 3. Multiple logistic regression analysis of the variables that influence on the occurrence of aspiration pneumonia in the patients with Parkinson disease (maxrescaled $\mathrm{R}^{2}=0.78$ )

\begin{tabular}{lcccc}
\hline & OR & $\mathbf{9 5 \%}$ CI & $\boldsymbol{\beta}$ & p-value \\
\hline S-E ADL & 0.89 & $0.80-0.96$ & -1.41 & $0.018^{*}$ \\
FDS & 1.10 & $1.01-1.20$ & 0.68 & $0.043^{*}$ \\
\hline
\end{tabular}

S-E ADL, Schwab and England activities of daily living scale; FDS, functional dysphagia scale; OR, odd ratio; CI, confidence interval; $\beta$, standardized estimates.

${ }^{*} \mathrm{p}<0.05$. 
aspiration pneumonia (Table 2).

According to the multiple logistic regression analysis, the S-E ADL (odds ratio, 0.89; $\mathrm{p}=0.018$ ) and the FDS (odds ratio, 1.10; $\mathrm{p}=0.043$ ) were independently associated with the occurrence of aspiration pneumonia in patients with Parkinson disease (max-rescaled $R^{2}=0.78$ ) (Table 3 ).

\section{DISCUSSION}

Dysphagia is commonly observed among patients with Parkinson disease and the resulting aspiration pneumonia can be fatal. Although the symptoms of dysphagia and a variety of other factors that cause aspiration pneumonia should be considered, aspiration pneumonia was observed significantly more frequently in Parkinson disease patients with a low functional status. This result has been discussed in a number of studies $[14,15]$.

A previous study reported that a prolonged pharyngeal transfer time [16] among patients with Parkinson disease is relevant to the occurrence of aspiration pneumonia. This is consistent with the results in this study, in which a significantly longer transfer time in the pharyngeal phase was observed among the patients with aspiration pneumonia. Moreover, in this study, abnormalities of the oral phase, the amount of residue in the pyriform sinus, and the coating of barium on the pharyngeal wall after swallowing were also different between the two groups. Among patients with Parkinson disease, this problem is caused by a delay in the food bolus' transit time in the oral and pharyngeal phases [21], as well as bradykinesia, rigidity, and tongue movement abnormalities [27,28]. The results of this study suggest that the frequency of aspiration pneumonia can increase due to a secondary process resulting from a decreased functional status and overall aggravation of the condition because of a poor oral diet.

Researchers previously found that a delay in the pharyngeal swallowing reflex is associated with aspiration pneumonia in Parkinson disease patients [29,30]. In this study, the abnormality in the pharyngeal phase in the FDS, which is a delayed triggering of the pharyngeal swallowing reflex, was apparent, although there was no statistical significance (Table 2). We assume that this indicates that the FDS abnormalities in the pharyngeal phase can be detected at a relatively high level, even in patients without aspiration pneumonia.
The PAS of the VFSS showed a significantly higher score among the Parkinson disease patients who had aspiration pneumonia, but it had limitations when reflecting dysphagia in movement abnormalities, which is an important area of discomfort for patients with Parkinson disease. In the multiple logistic regression analysis in this study, only the S-E ADL scale and the FDS were statistically significant, with odds ratios of 0.89 and 1.10, respectively. These were most relevant to the occurrence of aspiration pneumonia when the S-E ADL scale was lower and the FDS was higher (Table 3). As previously stated, patients with Parkinson disease suffer with swallowing difficulties, which are caused by bradykinesia and rigidity. Therefore, the status assessment provides a better reflection of swallowing movement and function than airway invasion for swallowing abnormalities among patients with Parkinson disease. Functional status can also influence the occurrence of aspiration pneumonia in patients with Parkinson disease.

This study had several limitations. First, the number of patients with aspiration pneumonia was small $(n=8)$. Second, we had a short follow-up period of 3 months. Although several studies had 6-month follow-up periods [16], follow-up over a longer term can have less significance in reflecting the condition and VFSS result a single point.

This study demonstrated that the occurrence of aspiration pneumonia among patients with Parkinson disease correlates with the FDS and the S-E ADL. Given that the FDS can quantitatively assess the functional problems of dysphagia, it is expected to be clinically effective in predicting the occurrence of aspiration pneumonia and selecting appropriate therapy options for dysphagia in patients with Parkinson disease.

\section{CONFLICT OF INTEREST}

No potential conflict of interest relevant to this article was reported.

\section{ACKNOWLEGDMENTS}

This study was supported by the research funds of Dong-A University. 


\section{REFERENCES}

1. Jankovic J. Parkinson's disease: clinical features and diagnosis. J Neurol Neurosurg Psychiatry 2008;79:36876.

2. Vaugoyeau M, Viel S, Assaiante C, Amblard B, Azulay JP. Impaired vertical postural control and proprioceptive integration deficits in Parkinson's disease. Neuroscience 2007;146:852-63.

3. Monte FS, da Silva-Junior FP, Braga-Neto P, Nobre e Souza MA, de Bruin VM. Swallowing abnormalities and dyskinesia in Parkinson's disease. Mov Disord 2005;20:457-62.

4. Lieberman AN, Horowitz L, Redmond P, Pachter L, Lieberman I, Leibowitz M. Dysphagia in Parkinson's disease. Am J Gastroenterol 1980;74:157-60.

5. Penner A, Druckerman LJ. Segmental spasms of the esophagus and their relation to Parkinsonism. Am J Dig Dis 1942;9:272-87.

6. Logemann JA, Gensler G, Robbins J, Lindblad AS, Brandt D, Hind JA, et al. A randomized study of three interventions for aspiration of thin liquids in patients with dementia or Parkinson's disease. J Speech Lang Hear Res 2008;51:173-83.

7. Marik PE, Kaplan D. Aspiration pneumonia and dysphagia in the elderly. Chest 2003;124:328-36.

8. Hoehn MM, Yahr MD. Parkinsonism: onset, progression and mortality. Neurology 1967;17:427-42.

9. Gorell JM, Johnson CC, Rybicki BA. Parkinson's disease and its comorbid disorders: an analysis of Michigan mortality data, 1970 to 1990 . Neurology 1994;44: 1865-8.

10. Shill H, Stacy M. Respiratory function in Parkinson's disease. Clin Neurosci 1998;5:131-5.

11. Fernandez HH, Lapane KL. Predictors of mortality among nursing home residents with a diagnosis of Parkinson's disease. Med Sci Monit 2002;8:CR241-6.

12. Mody L. Editorial commentary: preventing aspiration pneumonia in high-risk nursing home residents. Role of chlorhexidine-based oral care questioned again. Clin Infect Dis 2015;60:858-9.

13. Muller F. Oral hygiene reduces the mortality from aspiration pneumonia in frail elders. J Dent Res 2015;94(3 Suppl):14S-16S.

14. Kikutani T, Tamura F, Tashiro H, Yoshida M, Konishi K, Hamada R. Relationship between oral bacteria count and pneumonia onset in elderly nursing home residents. Geriatr Gerontol Int 2015;15:417-21.

15. Dang TT, Majumdar SR, Marrie TJ, Eurich DT. Recurrent pneumonia: a review with focus on clinical epidemiology and modifiable risk factors in elderly patients. Drugs Aging 2015;32:13-9.

16. Lin CW, Chang YC, Chen WS, Chang K, Chang HY, Wang TG. Prolonged swallowing time in dysphagic Parkinsonism patients with aspiration pneumonia. Arch Phys Med Rehabil 2012;93:2080-4.

17. Leopold NA, Kagel MC. Pharyngo-esophageal dysphagia in Parkinson's disease. Dysphagia 1997;12:118.

18. Robbins JA, Logemann JA, Kirshner HS. Swallowing and speech production in Parkinson's disease. Ann Neurol 1986;19:283-7.

19. Eadie MJ, Tyrer JH. Radiological abnormalities of the upper part of the alimentary tract in Parkinsonism. Australas Ann Med 1965;14:23-7.

20. Troche MS, Sapienza CM, Rosenbek JC. Effects of bolus consistency on timing and safety of swallow in patients with Parkinson's disease. Dysphagia 2008;23:2632.

21. Nagaya M, Kachi T, Yamada T. Effect of swallowing training on swallowing disorders in Parkinson's disease. Scand J Rehabil Med 2000;32:11-5.

22. Bird MR, Woodward MC, Gibson EM, Phyland DJ, Fonda D. Asymptomatic swallowing disorders in elderly patients with Parkinson's disease: a description of findings on clinical examination and videofluoroscopy in sixteen patients. Age Ageing 1994;23:251-4.

23. Han TR, Paik NJ, Park JW. Quantifying swallowing function after stroke: a functional dysphagia scale based on videofluoroscopic studies. Arch Phys Med Rehabil 2001;82:677-82.

24. Fahn S, Elton R. The unified Parkinson's disease rating scale. In: Fahn S, Marsden CD, Jenner P, Teycenne P, editors. Recent developments in Parkinson's disease. New York: Raven Press; 1986.

25. Logemann JA. Manual for the videofluorographic study of swallowing. 2nd ed. Austin: Pro-Ed; 1993.

26. Rosenbek JC, Robbins JA, Roecker EB, Coyle JL, Wood JL. A penetration-aspiration scale. Dysphagia 1996;11: 93-8.

27. Edwards LL, Quigley EM, Pfeiffer RF. Gastrointestinal dysfunction in Parkinson's disease: frequency and 
pathophysiology. Neurology 1992;42:726-32.

28. Bushmann M, Dobmeyer SM, Leeker L, Perlmutter JS. Swallowing abnormalities and their response to treatment in Parkinson's disease. Neurology 1989;39:130914.

29. Ertekin C, Tarlaci S, Aydogdu I, Kiylioglu N, Yuceyar N,
Turman AB, et al. Electrophysiological evaluation of pharyngeal phase of swallowing in patients with Parkinson's disease. Mov Disord 2002;17:942-9.

30. Stroudley J, Walsh M. Radiological assessment of dysphagia in Parkinson's disease. Br J Radiol 1991;64:8903. 